\title{
FLOW IN THE POROUS REGION BETWEEN SLOWLY ROTATING PROLATE SPHEROIDS
}

\author{
N. K. AGARWAL and SUNIL DATTA
}

(Received 6 February 1980)

(Revised 15 May 1980)

\begin{abstract}
In the present paper the flow in the porous region bounded by confocal prolate spheroids rotating slowly about the major axis is investigated by a singularity method.
\end{abstract}

\section{Introduction}

Narasimhacharyulu and Ramacharyulu [5] have recently investigated the steady flow in a porous region between two slowly rotating spheres employing Brinkman's [1] generalization of Darcy's law and using the method of separation of variables. This method when applied to the more complex geometry of spheroids leads to complicated expressions. A more simple approach is to follow the singularity method used earlier by Chwang and $\mathrm{Wu}[2,3]$ to study the slow motion of spheroids in a viscous fluid.

In this paper we too construct the solution to the problem of flow in the porous region between two prolate spheroids by using a suitable singularity distribution. The flow is discussed for large permeability coefficient $k$, and the results for the flow between concentric spheres and around a disk are obtained as limiting cases.

\section{Singular solution}

Brinkman's [1] equation governing the slow steady flow of viscous fluid in a porous medium are

$$
\mu \nabla^{2} \mathbf{V}-\frac{\mu}{k} \mathbf{V}+\mathbf{f}(\mathbf{x})=\nabla p
$$

OCopyright Australian Mathematical Society 1981 
and

$$
\nabla \cdot \mathbf{V}=0
$$

where $\mathbf{V}$ is the velocity field, $\mu$ the fluid viscosity, $k$ the permeability, $\mathrm{f}$ is the applied force and $\mathbf{x}$ the position vector. For rotatory motion $p$ is a constant and it is found convenient to take

$$
\mathbf{f}(\mathbf{x})=4 \pi \mu \nabla \times(\gamma \delta(\mathbf{x})),
$$

where $\mathbf{r}$ is a constant vector along the axis of rotation and $\delta(\mathbf{x})$ is the Dirac-delta function. Thus, by introducing the fundamental singular solution of equation (1), namely

$$
\mathbf{V}^{R}(\mathbf{x} ; \boldsymbol{\gamma})=\nabla \times\left\{\frac{\gamma}{R} \exp \left(-\frac{R}{\sqrt{ } k}\right)\right\}, \quad \text { where } R=|\mathbf{x}|,
$$

this reduces, when $k \rightarrow \infty$, to the "rotlet" used earlier by Chwang and $\mathrm{Wu}$ [3] and conveniently provides

$$
\mathbf{M}=-8 \pi \mu \gamma
$$

as the torque on a control volume containing the singular point.

We shall also need, corresponding to the "roton" of [3], the following fundamental solutions in the interior region:

$$
\mathbf{V}^{N}\left(\mathbf{x} ; \hat{\mathbf{e}}_{x}\right)=\frac{1}{R^{2}}\left\{\cosh \frac{R}{\sqrt{ } k}-\frac{V k}{R} \sinh \frac{R}{\sqrt{ } k}\right\} \hat{\mathbf{e}}_{x} \times \mathbf{x},
$$

where $\hat{\mathbf{e}}_{x}$ is the unit vector along the axis of rotation.

\section{Rotating prolate spheroids}

Let the prolate spheroids $S_{j}$, described by

$$
\frac{x^{2}}{a_{j}^{2}}+\frac{\omega^{2}}{b_{j}^{2}}=1 \quad \text { for } j=1,2,
$$

where $\omega^{2}=y^{2}+z^{2}, a_{j} \geqslant b_{j}$ and $a_{2}>a_{1}$, rotate about their major axes with angular velocities $\Omega_{j} \hat{\mathbf{e}}_{x}$. It should be noted that the common focal length $2 c$ and eccentricities $e_{j}$ are related as $c=a_{j} e_{j}$. The no-slip condition requires that

$$
\mathbf{V}=\Omega_{j} \hat{\mathbf{e}}_{x} \times \mathbf{x} \text { on } S_{j},
$$

where $S_{1}$ and $S_{2}$ represent the inner and outer body surfaces respectively.

It can be seen that the flow can be constructed by a distribution of fundamental singularities between the foci $x=-c$ and $x=c$. Thus, we take 


$$
\mathbf{V}=A \mathbf{V}^{N}+B_{0} \frac{\partial^{2}}{\partial x^{2}}\left(\mathbf{V}^{N}\right)+\int_{-c}^{c} \gamma(\xi) \mathbf{V}^{R}\left(\mathbf{x}-\xi ; \hat{\mathbf{e}}_{x}\right) d \xi
$$

where $\xi=\xi \hat{\mathbf{e}}_{x}$. For large values of $k$, we have the approximations

$$
\left.\begin{array}{l}
\mathbf{V}^{R}=\left(\frac{1}{R^{3}}-\frac{1}{2 k R}\right) \hat{\mathbf{e}}_{x} \times \mathbf{x}+O\left(\frac{1}{k^{2}}\right), \\
\mathbf{V}^{N}=\left(1+\frac{R^{2}}{10 k}\right) \hat{\mathbf{e}}_{x} \times \mathbf{x}+O\left(\frac{1}{k^{2}}\right), \\
\frac{\partial^{2}}{\partial x^{2}} \mathbf{V}^{N}=\left(1+\frac{R^{2}+2 x^{2}}{14 k}\right) \hat{\mathbf{e}}_{x} \times \mathbf{x}+o\left(\frac{1}{k^{2}}\right), \\
\gamma(\xi)=\gamma_{0}(\xi)+\frac{1}{k} \gamma_{2}(\xi) .
\end{array}\right\}
$$

Substituting the above, equation (9) becomes

$$
\begin{aligned}
\mathbf{v}= & {\left[A\left(1+\frac{R^{2}}{10 k}\right)+B_{0}\left(1+\frac{R^{2}+2 x^{2}}{14 k}\right)\right.} \\
& \left.+\int_{-c}^{c}\left\{\frac{r_{0}(\xi)}{R_{\xi}^{3}}+\frac{1}{k}\left(\frac{r_{2}(\xi)}{R_{\xi}^{3}}-\frac{r_{0}(\xi)}{2 R_{\xi}}\right)\right\} d \xi\right] \mathbf{e}_{x} \times \mathbf{x},
\end{aligned}
$$

where $R_{\xi}=\left\{(x-\xi)^{2}+\omega^{2}\right\}^{1 / 2}$.

We now set

$$
\begin{aligned}
& \gamma_{0}(\xi)=D_{0}\left(c^{2}-\xi^{2}\right) \\
& \gamma_{2}(\xi)=D_{2}\left(c^{2}-\xi^{2}\right)-D_{4} \xi^{2}\left(c^{2}-\xi^{2}\right) \\
& A=A_{0}+\frac{A_{2}}{k}
\end{aligned}
$$

Equation (11) reduces to

$$
\begin{aligned}
\mathbf{V}= & {\left[A_{0}\left(1+\frac{R^{2}}{10 k}\right)+\frac{A_{2}}{k}+B_{0}\left(1+\frac{2 x^{2}+R^{2}}{14 k}\right)\right.} \\
& +D_{0}\left(c^{2} I_{0,3}-I_{2,3}\right)-\frac{1}{k}\left\{\frac{D_{0}}{2}\left(c^{2} I_{0,1}-I_{2,1}\right)\right. \\
& \left.\left.-D_{2}\left(c^{2} I_{0,3}-I_{2,3}\right)+D_{4}\left(c^{2} I_{2,3}-I_{4,3}\right)\right\}\right] \mathbf{e}_{x} \times \mathbf{x},
\end{aligned}
$$

where

$$
I_{m, n}=\int_{-c}^{c} \frac{\xi^{m}}{\left\{(x-\xi)^{2}+\omega^{2}\right\}^{n / 2}} d \xi
$$


To determine the unknowns $A_{0}, A_{2}, B_{0}, D_{0}, D_{2}$ and $D_{4}$, we observe that the following relations are valid on $S_{j}$ :

$$
\left.\begin{array}{l}
R^{2}=\left(a_{j}^{2}-c^{2}\right)+e_{j}^{2} x^{2}, \\
\left(c^{2} I_{0,3}-I_{2,3}\right)=f_{j}, \\
\left(c^{2} I_{2,3}-I_{4,3}\right)=g_{j}+h_{j} x^{2} \\
\left(c^{2} I_{0,1}-I_{2,1}\right)=p_{j}+q_{j} x^{2},
\end{array}\right\}
$$

where

$$
\begin{aligned}
f_{j} & =\frac{2 e_{j}}{1-e_{j}^{2}}-L_{j}, \\
g_{j} & =a_{j}^{2}\left(\frac{3-e_{j}^{2}}{2} L_{j}-3 e_{J}\right), \\
h_{j} & =\frac{15 e_{j}-13 e_{j}^{3}}{1-e_{j}^{2}}-\frac{15-3 e_{j}^{2}}{2} L_{j}, \\
p_{j} & =a_{j}^{2}\left(\frac{1+e_{j}^{2}}{2} L_{j}-e_{j}\right), \\
q_{j} & =3 e_{j}-\frac{3-e_{j}^{2}}{2} L_{j}, \\
L_{j} & =\log \frac{1+e_{j}}{1-e_{j}} .
\end{aligned}
$$

Making use of above and applying boundary conditions (8), we have from equation (13),

$$
A_{0}=\left[5\left\{\left(2+e_{1}^{2}\right) h_{2}-\left(2+e_{2}^{2}\right) h_{1}\right\}\left(f_{1} \Omega_{2}-f_{2} \Omega_{1}\right)-35\left(q_{1} h_{2}-q_{2} h_{1}\right)\left(\Omega_{1}-\Omega_{2}\right)\right] P,
$$

$$
\begin{gathered}
A_{2}=\frac{1}{f_{1}-f_{2}}\left[\frac{1}{2}\left(f_{1} p_{2}-f_{2} p_{1}\right) D_{0}+\left(f_{1} g_{2}-f_{2} g_{1}\right) D_{4}\right. \\
+\left\{\left(\left(a_{2}^{2}-c^{2}\right) f_{1}-\left(a_{1}^{2}-c^{2}\right) f_{2}\right)\left(h_{1}-h_{2}\right)\left(f_{1} \Omega_{2}-f_{2} \Omega_{1}\right)\right. \\
\left.\left.-\left(a_{1}^{2}-a_{2}^{2}\right)\left(q_{1} h_{2}-q_{2} h_{1}\right)\left(\Omega_{1}-\Omega_{2}\right)\right\} P\right], \\
B_{0}=\left[35\left(q_{1} h_{2}-q_{2} h_{1}\right)\left(\Omega_{1}-\Omega_{2}\right)-7\left(e_{1}^{2} h_{2}-e_{2}^{2} h_{1}\right)\left(f_{1} \Omega_{2}-f_{2} \Omega_{1}\right)\right] P, \\
D_{0}=\frac{\Omega_{1}-\Omega_{2}}{f_{1}-f_{2}},
\end{gathered}
$$




$$
\begin{aligned}
D_{2}= & \frac{1}{f_{1}-f_{2}}\left[\frac{1}{2}\left(p_{1}-p_{2}\right) D_{0}+\left(g_{1}-g_{2}\right) D_{4}+\left(a_{1}^{2}-a_{2}^{2}\right)\right. \\
& \left.\times\left\{\left(h_{1}-h_{2}\right)\left(f_{1} \Omega_{2}-f_{2} \Omega_{1}\right)+\left(q_{1} h_{2}-q_{2} h_{1}\right)\left(\Omega_{1}-\Omega_{2}\right)\right\} P\right], \\
D_{4}= & {\left[\left(\left(5-e_{2}^{2}\right) q_{1}-\left(5-e_{1}^{2}\right) q_{2}\right)\left(\Omega_{1}-\Omega_{2}\right)-\left(e_{1}^{2}-e_{2}^{2}\right)\left(f_{1} \Omega_{2}-f_{2} \Omega_{1}\right)\right] P, }
\end{aligned}
$$

where $P=\left[2\left\{\left(5-e_{1}^{2}\right) h_{2}-\left(5-e_{2}^{2}\right) h_{1}\right\}\left(f_{1}-f_{2}\right)\right]^{-1}$.

The torque $\mathbf{M}$ on the inner spheroid is given by

$$
\begin{aligned}
\mathbf{M}= & -8 \pi \mu \hat{\mathbf{e}}_{x} \int_{-c}^{c} r(\xi) d \xi, \\
= & -\frac{32 \pi \mu c^{3} \hat{\mathbf{e}}_{x}}{3\left(f_{1}-f_{2}\right)}\left[\left\{1+\frac{a_{1}^{2}\left(1+3 e_{1}^{2}\right) L_{1}-2 a_{1}^{2} e_{1}+a_{1}^{2}\left(1-e_{1}^{2}\right) f_{2}-4 c^{2} L_{2}}{10 k\left(f_{1}-f_{2}\right)}\right\} \Omega_{1}\right. \\
& \left.-\left\{1-\frac{a_{2}^{2}\left(1+3 e_{2}^{2}\right) L_{2}-2 a_{2}^{2} e_{2}+a_{2}^{2}\left(1-e_{2}^{2}\right) f_{1}-4 c^{2} L_{1}}{10 k\left(f_{1}-f_{2}\right)}\right\} \Omega_{2}\right] .
\end{aligned}
$$

\section{Special situations}

\section{Non-porous medium}

Taking the limit as $k \rightarrow \infty$, equations (13) and (17) reduce respectively to

$$
\mathbf{V}=\left[\frac{f_{1} \Omega_{2}-f_{2} \Omega_{1}}{f_{1}-f_{2}}+\frac{\Omega_{1}-\Omega_{2}}{f_{1}-f_{2}}\left(c^{2} I_{0,3}-I_{2,3}\right)\right] \hat{\mathbf{e}}_{x} \times \mathbf{x}
$$

and

$$
\mathbf{M}=-\frac{32}{3} \pi \mu c^{3} \frac{\Omega_{1}-\Omega_{2}}{f_{1}-f_{2}} \hat{\mathbf{e}}_{x},
$$

which are the same as those obtained by Chwang and $\mathrm{Wu}$ [3].

\section{Prolate spheroid in an infinite medium}

The flow induced by a prolate spheroid rotating about its major axis in an infinite porous medium can be realized by letting $a_{2} \rightarrow \infty$ and $e_{2} \rightarrow 0$. Thus we have from equations (13) and (17)

$$
\begin{aligned}
\mathbf{V}= & \frac{\Omega_{1}}{f_{1}}\left[\left(c^{2} I_{0,3}-I_{2,3}\right)+\frac{1}{2 k}\left\{\frac{p_{1} h_{1}-q_{1} g_{1}}{f_{1} h_{1}}\left(c^{2} I_{0,3}-I_{2,3}\right)\right.\right. \\
& \left.\left.+\frac{q_{1}}{h_{1}}\left(c^{2} I_{2,3}-I_{4,3}\right)-\left(c^{2} I_{0,1}-I_{2,1}\right)\right\}\right] \hat{\mathbf{e}}_{x} \times \mathbf{x}
\end{aligned}
$$


and

$$
\mathbf{M}=-\frac{32}{3 f_{1}} \pi \mu c^{3} \Omega_{1} \hat{\mathbf{e}}_{x}\left(1+\frac{a_{1}^{2}\left(1+3 e_{1}^{2}\right) L_{1}-2 a_{1}^{2} e_{1}}{10 k f_{1}}\right)
$$

As $k \rightarrow \infty$ in the above expressions, we get

$$
\mathrm{V}=\Omega_{1}\left(c^{2} I_{0,3}-I_{2,3}\right)\left(\frac{2 e_{1}}{1-e_{1}^{2}}-L_{1}\right)^{-1} \hat{\mathbf{e}}_{x} \times \mathbf{x}
$$

and

$$
\mathbf{M}=-\frac{32}{3} \pi \mu c^{3} \Omega_{1} \hat{e}_{x}\left(\frac{2 e_{1}}{1-e_{1}^{2}}-L_{1}\right)^{-1},
$$

which are the same as those obtained in [2].

\section{Rotating spheres}

Flow in a porous region between two slowly rotating spheres is obtained by letting $e_{1} \rightarrow 0$ and $e_{2} \rightarrow 0$ in equation (13), thereby giving

$$
\begin{aligned}
\mathbf{V}= & {\left[a_{2}^{3} \Omega_{2}\left\{\left(r^{3}-a_{1}^{3}\right)+\frac{1}{10 k}\left(r-a_{1}\right)^{3}\left(r^{2}+3 a_{1} r+a_{1}^{2}\right)\right\}\right.} \\
& \left.-a_{1}^{3} \Omega_{1}\left\{\left(r^{3}-a_{2}^{3}\right)+\frac{1}{10 k}\left(r-a_{2}\right)^{3}\left(r^{2}+3 a_{2} r+a_{2}^{2}\right)\right\}\right] \\
& \times r^{-3}\left[\left(a_{2}^{3}-a_{1}^{3}\right)+\frac{1}{10 k}\left(a_{2}-a_{1}\right)^{3}\left(a_{2}^{2}+3 a_{1} a_{2}+a_{1}^{2}\right)\right]^{-1} \hat{\mathbf{e}}_{x} \times \mathbf{x},
\end{aligned}
$$

which corresponds to the expressions obtained by [5]. As $k \rightarrow \infty$ the above equation reduces to

$$
\mathbf{V}=\frac{r^{-3}}{\left(a_{2}^{3}-a_{1}^{3}\right)}\left[a_{2}^{3} \Omega_{2}\left(r^{3}-a_{1}^{3}\right)-a_{1}^{3} \Omega_{1}\left(r^{3}-a_{2}^{3}\right)\right] \hat{\mathbf{e}}_{x} \times \mathbf{x}
$$

which is the well known slow flow between concentric spheres. Again letting $e_{1} \rightarrow 0$ and $e_{2} \rightarrow 0$ in (17), we obtain

$$
\mathbf{M}=\frac{8 \pi \mu a_{1}^{3} a_{2}^{3}\left[\left(1+\frac{a_{1}^{2}}{10 k}\right) \Omega_{2}-\left(1+\frac{a_{2}^{2}}{10 k}\right) \Omega_{1}\right]}{\left(a_{2}^{3}-a_{1}^{3}\right)+\frac{1}{10 k}\left(a_{2}-a_{1}\right)^{3}\left(a_{2}^{2}+3 a_{1} a_{2}+a_{1}^{2}\right)} \hat{\mathbf{e}}_{x} .
$$

If we let $a_{2} \rightarrow \infty$, the net couple on a sphere rotating in an infinite porous medium is obtained as

$$
\mathbf{M}=-8 \pi \mu \Omega_{1} a_{1}^{3}\left(1+\frac{a_{1}^{2}}{2 k}\right) \hat{\mathbf{e}}_{x}
$$


It is seen that $|\mathbf{M}|$, as obtained from equation (27), differs from the moment $M_{a_{1}}=-8 \pi \mu \Omega_{1} a_{1}^{3}\left(1+a_{1}^{2} / 3 k\right)$, given by equation (17) in [5] (after replacing $a$ by $a_{1}, \Omega_{a}$ by $\Omega_{1}$ and making the approximation $1+\sqrt{ } k / a_{1} \sim \vee k / a_{1}$ for large $k$ ), because the latter is only the contribution of viscous stress. The contribution of the permeability term $\left(\mu V / k\right.$ of equation (1)) is calculated to be $-(4 \pi / 3 k) \mu \Omega_{1} a_{1}^{5}$ and, on addition to the viscous stress part, the total couple is again recovered.

\section{Oblate spheroid and circular disk}

The results for an oblate spheroid can be deduced from above relations by replacing $c$ by $-i c$ and $e_{j}$ by $-i e_{j} /\left(1-e_{j}^{2}\right)^{1 / 2}$. The interesting case of a circular disk rotating in an infinite medium is obtained by letting $e_{2} \rightarrow 0, a_{2} \rightarrow 0$ and $e_{1} \rightarrow 1$. Thus, from equation (17) we have

$$
\mathbf{M}=-\frac{32}{3} \pi \mu a_{1}^{3} \Omega_{1}\left(1+\frac{3 a_{1}^{2}}{10 k}\right) \hat{\mathbf{e}}_{x} .
$$

It should be observed that equation (1) also governs the rotary oscillations in Stokes flow when the $\mu \mathrm{V} / k$ term is interpreted as the inertia term with the time factor $e^{i w t}$ suppressed. The results deduced in this paper also provide the results for the corresponding rotary oscillation problems. But it is seen that the total couple as given by (25) differs from that given in [4] (equation 66, page 265) where the coefficient of $\beta^{2}\left(=a_{1}^{2} / k\right)$ is $\frac{1}{5}$ instead of $3 / 10$. This discrepancy can be accounted for by the contribution of the inertia term, as in the case of the sphere.

\section{References}

[1] H. C. Brinkman, "A calculation of the viscous force exerted by a flowing fluid on a dense swarm of particles", Appl. Sc. Res. Al (1947), 27-34.

[2] A. T. Chwang and T. Y. Wu, "Hydrodynamics of low-Reynolds number flow", J. Fluid Mech., Pt. I 63 (1974), 607-622.

[3] A. T. Chwang and T. Y. Wu, "Hydrodynamics of low-Reynolds number flow", J. Fluid Mech., Part II 67 (1975), 787-815.

[4] R. P. Kanwal, Linear integral equations (Academic Press, 1971).

[5] V. Narasimhacharyulu and N. Ch. Pattabhi Ramachyarulu, "Steady flow in a porous region between two slowly rotating spheres", J. I. I. Sc. 60 (1978), 247-252.

Department of Mathematics and Astronomy

Lucknow University

Lucknow 226007

India 